\title{
Solutions for impulsive fractional pantograph differential equation via generalized anti-periodic boundary condition
}

\author{
Idris Ahmed ${ }^{1,2,3}$ (D), Poom Kumam ${ }^{2,5^{*}}$ (B), Jamilu Abubakar ${ }^{1,2,4}$ (B), Piyachat Borisut ${ }^{1,2}$ (D) and \\ Kanokwan Sitthithakerngkiet ${ }^{6}$ (D)
}

\author{
"Correspondence: \\ poom.kumam@mail.kmutt.ac.th \\ ${ }^{2}$ Center of Excellence in Theoretical \\ and Computational Science \\ (TaCS-CoE), Science Laboratory \\ Building, King Mongkut's University \\ of Technology Thonburi (KMUTT), \\ 126 Pracha-Uthit Road, Bang Mod, \\ Thrung Khru, Bangkok, 10140, \\ Thailand \\ ${ }^{5}$ Department of Medical Research, \\ China Medical University Hospital, \\ China Medical University, Taichung, \\ 40402, Taiwan \\ Full list of author information is \\ available at the end of the article
}

\begin{abstract}
This study investigates the solutions of an impulsive fractional differential equation incorporated with a pantograph. This work extends and improves some results of the impulsive fractional differential equation. A differential equation of an impulsive fractional pantograph with a more general anti-periodic boundary condition is proposed. By employing the well-known fixed point theorems of Banach and Krasnoselskii, the existence and uniqueness of the solution of the proposed problem are established. Furthermore, two examples are presented to support our theoretical analysis.
\end{abstract}

Keywords: Pantograph differential equation; Impulsive; Anti-periodic condition; Fixed point theorems

\section{Introduction}

Fractional differential equations are generally applicable in many fields such as chemistry, mechanics, fluid systems, electronics, electromagnetic and other fields; for an overview, the reader should see the literature on fractional differential equations, e.g., $[3,4,12,17,21$, $24,26,33-36,39]$ and the references therein. Fractional and impulsive differential equations were used as a powerful method to gain insight into certain emerging problems from various science and engineering fields [32, 40, 46]. In particular, much attention has been given to the theoretical studies such as existence, uniqueness, and stability of analytical solutions, in recent years (we refer, for example, to [1, 2, 6, 14-16, 38, 43]).

Several works on boundary value problems for an impulsive differential equation with anti-periodic boundary conditions were conducted, and results on the existence of solutions for mixed-type fractional integro-differential equation were established (see, e.g., $[5,19,30,42,51,52,54,55])$. More recently, the theory of existence, uniqueness, and stability analysis for impulsive fractional differential equations with different kinds of fractional operators and initial/boundary conditions has attracted the attention of many researchers; for an overview of the literature, we refer the reader to [8-11, 49, 50]. For example, in [47] Wang and Lin investigated the impulsive fractional anti-periodic boundary value prob-

(c) The Author(s) 2020. This article is licensed under a Creative Commons Attribution 4.0 International License, which permits use, sharing, adaptation, distribution and reproduction in any medium or format, as long as you give appropriate credit to the original author(s) and the source, provide a link to the Creative Commons licence, and indicate if changes were made. The images or other third party material in this article are included in the article's Creative Commons licence, unless indicated otherwise in a credit line to the material. If material is not included in the article's Creative Commons licence and your intended use is not permitted by statutory regulation or exceeds the permitted use, you will need to obtain permission directly from the copyright holder. To view a copy of this licence, visit http://creativecommons.org/licenses/by/4.0/. 
lem with constant coefficients. Recently, motivated by [47], Zuo et al. [56] investigated the existence results for an equation with impulsive and anti-periodic boundary conditions described by:

$$
\left\{\begin{array}{l}
{ }^{c} D_{0+}^{q} u(t)+\lambda u(t)=f(t, u(t), T u(t), S u(t)), \quad t \in J^{\prime}=J \backslash\left\{t_{1}, \ldots, t_{m}\right\}, \\
\left.\Delta u\right|_{t=t_{k}}=I_{k}\left(u\left(t_{k}\right)\right), \quad k=1,2, \ldots, m, \\
u(0)=-u(1),
\end{array}\right.
$$

where ${ }^{c} D^{q}$ is the Caputo fractional derivative of order $q \in(0,1), \lambda>0, I_{k} \in \mathbb{R}, 0=t_{0}<t_{1}<$ $\cdots<t_{m}<t_{m+1}=1, f \in C\left(J \times \mathbb{R}^{3}, \mathbb{R}\right), J=[0,1], \mathbb{R}$ is the set of real numbers, $\left.\Delta u\right|_{t=t_{k}}$ denotes the jump of $u(t)$ at $t=t_{k}, S$ and $T$ are linear operators. Under Lipschitz and nonlinear growth conditions, they established sufficient conditions for the existence and uniqueness of a solution to (1.1) using Banach mapping principle and Krasnoselskii's fixed point theorem.

On the other hand, in the deterministic situation there is a very special delay differential equation known as the pantograph equation given by

$$
z^{\prime}(\tau)=\alpha z(\tau)+\beta z(\lambda \tau), \quad 0 \leq \tau \leq T
$$

where $0<\lambda<1$. It is used in various fields of applied and pure mathematics, such as number theory, probability, dynamic system, and quantum mechanics. In particular, an important studies were conducted on the properties of both the analytical and numerical solutions of this equation (see $[18,22,23,31]$ ), also recently multi-pantograph and generalized nonlinear multi-pantograph equations were studied in [29, 37, 53]. Owing to the increasing interest and importance of this equation, Balachandran et al. [13] established the solution of abstract fractional pantograph equation via fractional calculus techniques and fixed point method. They consider the following equation:

$$
\left\{\begin{array}{l}
{ }^{c} D_{0+}^{p} y(t)=f(t, y(t), y(\lambda t)), \quad t \in J \\
y(0)=y_{0}
\end{array}\right.
$$

where $0<p<1,0<\lambda<1$, and $f: J \times X \times X \rightarrow X$ is a continuous function.

In this paper, motivated by $[13,47,56]$, we consider the following impulsive fractional pantograph differential equation:

$$
\left\{\begin{array}{c}
{ }^{c} D_{0+}^{\alpha} x(t)+\lambda x(t)=f(t, x(t), x(\gamma t)), \\
t \in J^{*}=J \backslash\left\{t_{1}, \ldots, t_{k}\right\}, 0<\alpha<1,0<\gamma<1, \\
\left.\Delta x\right|_{t=t_{m}}(0)=I_{m}\left(x\left(t_{m}\right)\right), \quad m=1,2, \ldots, k, \\
a x(0)+b x(1)=0, \quad a \geq b>0,
\end{array}\right.
$$

where ${ }^{c} D^{\alpha}$ is the Caputo fractional derivative of order $\alpha, 0=t_{0}<t_{1}<\cdots<t_{k}<t_{k+1}=1$, $f \in C\left(J \times \mathbb{R}^{2}, \mathbb{R}\right), J=[0,1],\left.\Delta x\right|_{t=t_{m}}=x\left(t_{m}^{+}\right)-x\left(t_{m}^{-}\right)$, with $x\left(t_{m}^{+}\right)$and $x\left(t_{m}^{-}\right)$representing the right and left limits of $x(t)$ at $t=t_{m}$.

The main aim of this paper is to establish the existence and uniqueness of solutions for the boundary value problem (1.3), by using the contraction principle of Banach and the 
fixed point theorem of Krasnoselskii. Presently, different techniques have been extensively applied in obtaining solutions to the impulsive fractional differential equations (see, e.g., $[20,41,44])$. However, in this article, we adopt the solution approach used in [47] to solve the impulsive fractional equation (1.3).

We highlight the main contributions of this paper as follows:

- We consider the impulsive pantograph fractional differential equation.

- We consider more general anti-periodic boundary value problems with constant coefficients.

In general, this paper contributes toward the development of qualitative analysis of fractional differential equations.

This paper is organized as follows: the statement of the problem, preliminaries, and some useful lemmas that will be required for the later sections are presented in Sect. 2. In Sect. 3, we prove the existence and uniqueness of solutions for problem (1.3) via Banach and Krasnoselskii's fixed point theorems with some illustrative examples. Conclusions on our findings are presented in the last section.

\section{Preliminaries and lemmas}

Let $J_{0}=\left(0, t_{1}\right], J_{1}=\left(t_{1}, t_{2}\right], \ldots, J_{k-1}, J_{k}=\left(t_{k}, 1\right]$, and $P C(J, \mathbb{R})=\left\{x: J \rightarrow \mathbb{R}: x \in C\left(J_{m}, \mathbb{R}\right)\right\}$, where $m=0,1,2, \ldots, k, x\left(t_{m}^{+}\right)$and $x\left(t_{m}^{-}\right)$exist, $m=1,2, \ldots, k$, is a space of continuous realvalued functions on the interval $J$, and $x\left(t_{m}^{-}\right)=x\left(t_{m}\right)$.

Then, clearly, $P C(J, \mathbb{R})$ is a Banach space with the norm $\|x\|_{P C}=\sup \{|x(t)|: t \in J\}$, and let the norm of a measurable function $\varphi: J \rightarrow \mathbb{R}$ be defined by:

$$
\|\varphi\|_{L^{p}(J)}=\left\{\begin{array}{l}
\left(\int_{J}|\varphi(t)|^{p} d t\right)^{1 / p}, \quad 1 \leq p<\infty, \\
\inf _{\operatorname{mes}(\bar{J})=0}\left\{\sup _{t \in J \bar{V}}|x(t)|\right\}, \quad p=\infty .
\end{array}\right.
$$

Then $L^{p}(J, \mathbb{R})$ is a Banach space of Lebesque-measurable functions with $\|\varphi\|_{\left.L^{p}()\right)}<\infty$.

Definition 2.1 (see [26]) The fractional integral of order $\rho$ with the lower limit zero for a function $g$ is defined as

$$
I_{0+}^{\rho} g(t)=\frac{1}{\Gamma(\rho)} \int_{0}^{t}(t-\tau)^{\rho-1} f(\tau) d \tau, \quad \rho>0, n \in \mathbb{N}
$$

provided the right-hand side is pointwise defined on $[0, \infty)$, where $\Gamma(\cdot)$ denotes the Gamma function.

Definition 2.2 (see [26]) The Riemann-Liouville derivative of order $\rho$ with the lower limit zero for a function $g$ is defined as

$$
{ }^{L} D_{0+}^{\rho} g(t)=\frac{1}{\Gamma(n-\rho)} \frac{d^{n}}{d t^{n}} \int_{0}^{t}(t-\tau)^{n-\rho-1} g(\tau) d \tau, \quad \rho>0, n-1<\rho<n,
$$

provided the function $g$ is absolutely continuous up to order $(n-1)$ derivatives, where $\Gamma(\cdot)$ denotes the Gamma function. 
Definition 2.3 (see [26]) The Caputo derivative of order $\rho>0$ with the lower limit zero for a function $g$ is defined as

$$
{ }^{c} D_{0++}^{\rho} g(t)=\frac{1}{\Gamma(n-\rho)} \frac{d^{n}}{d t^{n}} \int_{0}^{t}(t-\tau)^{n-\rho-1}\left(g(\tau)-\sum_{l=0}^{n-1} \frac{s^{l}}{l !} g^{(l)}(0)\right) d \tau, \quad n=[\rho]+1, n \in \mathbb{N}
$$

provided the function $g:[0, \infty) \rightarrow \mathbb{R}$, where $\Gamma(\cdot)$ denotes the Gamma function.

Remark 2.4 (see [28]) If $g \in C^{n}[0,+\infty$ ), then

$$
{ }^{c} D_{0+}^{\rho} g(t)=\frac{1}{\Gamma(n-\rho)} \int_{0}^{t}(t-\tau)^{n-\rho-1} g^{(n)}(\tau) d \tau=I^{n-\rho} g^{(n)}(t), \quad t>0, n=[\rho]+1 .
$$

Since in this paper we deal with an impulsive problem, Definition 2.3 is appropriate.

Definition 2.5 A function $x \in P C(J, \mathbb{R})$ is said to be a solution of problem (1.3) if it satisfies the equation ${ }^{c} D_{0_{+}}^{\alpha} x(t)+\lambda x(t)=f(t, x(t), x(\gamma t))$ a.e. on $J^{*}$ and the conditions $\left.\Delta x\right|_{t=t_{m}}(0)=$ $I_{m}\left(x\left(t_{m}\right)\right), m=1,2, \ldots, k$, and $a x(0)+b x(1)=0$, with $a \geq b>0$.

Lemma 2.6 (see [45]) The nonnegative functions $\mathrm{E}_{\alpha}$ and $\mathrm{E}_{\alpha, \alpha}$ given by

$$
\mathrm{E}_{\alpha}(u)=\sum_{m=0}^{\infty} \frac{u^{m}}{\Gamma(\alpha m+1)}, \quad \mathrm{E}_{\alpha, \alpha}(u)=\sum_{m=0}^{\infty} \frac{u^{m}}{\Gamma(\alpha m+\alpha)},
$$

have the following properties:

(1) For any $\lambda>0$ and $t \in J$,

$$
\mathrm{E}_{\alpha}\left(-t^{\alpha} \lambda\right) \leq 1, \quad \mathrm{E}_{\alpha, \alpha}\left(-t^{\alpha} \lambda\right) \leq \frac{1}{\Gamma(\alpha)}
$$

Moreover, $\mathrm{E}_{\alpha}(0)=1, \mathrm{E}_{\alpha, \alpha}(0)=\frac{1}{\Gamma(\alpha)}$.

(2) For any $\lambda>0$ and $t_{1}, t_{2} \in J$,

$$
\begin{aligned}
& \mathrm{E}_{\alpha}\left(-t_{2}^{\alpha} \lambda\right) \rightarrow \mathrm{E}_{\alpha}\left(-t_{1}^{\alpha} \lambda\right) \quad \text { as } t_{2} \rightarrow t_{1}, \\
& \mathrm{E}_{\alpha, \alpha}\left(-t_{2}^{\alpha} \lambda\right) \rightarrow \mathrm{E}_{\alpha, \alpha}\left(-t_{1}^{\alpha} \lambda\right) \quad \text { as } t_{2} \rightarrow t_{1} .
\end{aligned}
$$

(3) For any $\lambda>0$ and $t_{1}, t_{2} \in J$ such that $t_{1} \leq t_{2}$,

$$
\mathrm{E}_{\alpha}\left(-t_{2}^{\alpha} \lambda\right) \leq \mathrm{E}_{\alpha}\left(-t_{1}^{\alpha} \lambda\right), \quad \mathrm{E}_{\alpha, \alpha}\left(-t_{2}^{\alpha} \lambda\right) \leq \mathrm{E}_{\alpha, \alpha}\left(-t_{1}^{\alpha} \lambda\right)
$$

Lemma 2.7 (see [27]) Let $P$ be a closed, convex, and nonempty subset of a Banach space $X$, and let $F_{1}, F_{2}$ be operators such that:

(1) $F_{1} x+F_{2} y \in M$ whenever $x, y \in P$,

(2) $F_{1}$ is compact and continuous,

(3) $F_{2}$ is a contraction mapping.

Then there exists $z \in P$ such that $z=F_{1} z+F_{2} z$. 
Lemma 2.8 (see [48]) Let $X$ be a Banach space, and let $J=[0, T]$. Suppose that $\mathcal{W} \subset$ $P C(J, X)$ satisfies the following conditions:

(1) $\mathcal{W}$ is a uniformly bounded subset of $P C(J, X)$,

(2) $\mathcal{W}$ is equicontinuous in $\left(t_{m}, t_{m+1}\right), m=0,1, \ldots, k$, where $t_{0}=0, t_{k+1}=T$,

(3) Its $t$-sections $\mathcal{W}(t)=\left\{x(t): x \in \mathcal{W}, t \in J\left\{t_{1}, \ldots, t_{k}\right\}\right\}, \mathcal{W}\left(t_{m}^{+}\right)=\left\{x\left(t_{m}^{+}\right): x\left(t_{m}^{+}\right): x \in W\right\}$, and $\mathcal{W}\left(t_{m}^{-}\right)=\left\{x\left(t_{m}^{-}\right): x\left(t_{m}^{-}\right): x \in W\right\}$ are relatively compact subsets of $X$.

Then $\mathcal{W}$ is a relatively compact subset of $P C(J, X)$.

Lemma 2.9 (see [47]) Let $g: J \rightarrow \mathbb{R}$ be a continuous function. The function $u$ given by

$$
u(t)=\left\{\begin{array}{l}
\frac{-E_{q}(-\lambda) E_{q}\left(-t^{q} \lambda\right)}{1+E_{q}(-\lambda)} \sum_{i=1}^{m} \frac{y_{i}}{E_{q}\left(-t_{i}^{q} \lambda\right)}+\int_{0}^{t}(t-s)^{q-1} E_{q, q}\left(-(t-s)^{q} \lambda\right) g(s) d s \\
\quad-\frac{E_{q}\left(-t^{q} \lambda\right)}{1+E_{q}(-\lambda)} \int_{0}^{1}(1-s)^{q-1} E_{q, q}\left(-(1-s)^{q} \lambda\right) g(s) d s, \quad t \in J_{0}, \\
\frac{E_{q}\left(-t^{q} \lambda\right)}{1+E_{q}(-\lambda)}\left\{\sum_{i=1}^{m} \frac{y_{i}}{E_{q}\left(-t_{i}^{q} \lambda\right)}-\int_{0}^{1}(1-s)^{q-1} E_{q, q}\left(-(1-s)^{q} \lambda\right) g(s) d s\right\} \\
\quad-E_{q}\left(-t^{q} \lambda\right) \sum_{j=k+1}^{m} \frac{y_{j}}{E_{q}\left(-t_{j}^{q} \lambda\right)} \\
\quad+\int_{0}^{t}(t-s)^{q-1} E_{q, q}\left(-(t-s)^{q} \lambda\right) g(s) d s, \quad t \in J_{m}, m=1,2, \ldots, k-1, \\
\frac{E_{q}\left(-t^{q} \lambda\right)}{1+E_{q}(-\lambda)}\left\{\sum_{i=1}^{m} \frac{y_{i}}{E_{q}\left(-t_{i}^{q} \lambda\right)}-\int_{0}^{1}(1-s)^{q-1} E_{q, q}\left(-(1-s)^{q} \lambda\right) g(s) d s\right\} \\
\quad+\int_{0}^{t}(t-s)^{q-1} E_{q, q}\left(-(t-s)^{q} \lambda\right) g(s) d s, \quad t \in J_{k},
\end{array}\right.
$$

is a solution of the impulsive problem

$$
\left\{\begin{array}{l}
{ }^{c} D_{0+}^{q} u(t)+\lambda u(t)=g(t), \quad t \in J^{*}, \\
\left.\Delta u\right|_{t=t_{m}}(0)=y_{m}, \quad m=1,2, \ldots, k, \\
u(0)+u(1)=0 .
\end{array}\right.
$$

It follows from Lemma 2.9 and by using the boundary condition $a x(0)+b x(1)=0$ that the solution of (1.3) can be expressed by

$$
u(t)=\left\{\begin{array}{l}
\frac{-E_{\alpha}(-\lambda) E_{\alpha}\left(-t^{\alpha} \lambda\right)}{1+E_{\alpha}(-\lambda)} \sum_{i=1}^{k} \frac{I_{i}\left(x\left(t_{i}\right)\right)}{E_{\alpha}\left(-t_{i}^{\alpha} \lambda\right)} \\
\quad+\int_{0}^{t}(t-s)^{\alpha-1} E_{\alpha, \alpha}\left(-(t-s)^{\alpha} \lambda\right) f(s, x(s), x(\gamma s) d s \\
\quad-\frac{E_{\alpha}\left(-t^{\alpha} \lambda\right)}{1+\kappa E_{\alpha}(-\lambda)} \kappa \int_{0}^{1}(1-s)^{\alpha-1} E_{\alpha, \alpha}\left(-(1-s)^{\alpha} \lambda\right) f\left(s, x(s), x(\gamma s) d s, \quad t \in J_{0},\right. \\
\frac{E_{\alpha}\left(-t^{\alpha} \lambda\right)}{1+\kappa E_{\alpha}(-\lambda)}\left\{\sum_{i=1}^{k} \frac{I_{i}\left(x\left(t_{i}\right)\right)}{E_{\alpha}\left(-t_{i}^{\alpha} \lambda\right)}\right. \\
\left.\quad-\kappa \int_{0}^{1}(1-s)^{\alpha-1} E_{\alpha, \alpha}\left(-(1-s)^{\alpha} \lambda\right) f(s, x(s), x(\gamma s)) d s\right\} \\
\quad-E_{\alpha}\left(-t^{\alpha} \lambda\right) \sum_{j=m+1}^{k} \frac{I_{j}\left(x\left(t_{j}\right)\right)}{E_{\alpha}\left(-t_{j}^{\alpha} \lambda\right)} \\
\quad+\int_{0}^{t}(t-s)^{\alpha-1} E_{\alpha, \alpha}\left(-(t-s)^{\alpha} \lambda\right) f(s, x(s), x(\gamma s)) d s, \\
\quad t \in J_{m}, m=1,2, \ldots, k-1, \\
\frac{E_{\alpha}\left(-t^{\alpha} \lambda\right)}{1+\kappa E_{\alpha}(-\lambda)}\left\{\sum_{i=1}^{k} \frac{I_{i}\left(x\left(t_{i}\right)\right)}{E_{\alpha}\left(-t_{i}^{\alpha} \lambda\right)}\right. \\
\left.\quad-\kappa \int_{0}^{1}(1-s)^{\alpha-1} E_{\alpha, \alpha}\left(-(1-s)^{\alpha} \lambda\right) f(s, x(s), x(\gamma s)) d s\right\} \\
\quad+\int_{0}^{t}(t-s)^{\alpha-1} E_{\alpha, \alpha}\left(-(t-s)^{\alpha} \lambda\right) f(s, x(s), x(\gamma s)) d s, \quad t \in J_{k},
\end{array}\right.
$$

where $\kappa=\frac{b}{a}$. 


\section{Main results}

Theorem 3.1 Consider the following hypotheses:

$\left(C_{1}\right)$ Function $f \times J \times \mathbb{R}^{2} \rightarrow \mathbb{R}$ is continuous and there exists a constant $L_{1}>0$ such that

$$
|f(t, x, y)-f(t, u, v)| \leq L_{1}(|x-u|+|y-v|),
$$

for all $t \in J, x, y, u, v \in \mathbb{R}$.

$\left(C_{2}\right)$ There exists a positive constant $L_{2}$ such that

$$
\left|I_{m}(x)-I_{m}(y)\right| \leq L_{2}|x-y|, \quad \text { for all } x, y \in \mathbb{R}, m=1,2, \ldots, k \text {. }
$$

$\left(C_{3}\right)$

$$
\eta=\frac{(2+\kappa)}{\left|1+\kappa E_{\alpha}(-\lambda)\right|}\left(\sum_{i=1}^{k} \frac{L_{2}}{\left|E_{\alpha}\left(-t_{i}^{\alpha} \lambda\right)\right|}+\frac{(4 \kappa+2) L_{1}}{(2+\kappa) \Gamma(\alpha+1)}\right)<1 .
$$

Then the boundary value problem (1.3) has a unique solution.

Proof Define a mapping $T: P C(J, \mathbb{R}) \rightarrow P C(J, \mathbb{R})$ by

$$
\begin{aligned}
(T x)(t)= & \frac{E_{\alpha}\left(-t^{\alpha} \lambda\right)}{1+\kappa E_{\alpha}(-\lambda)}\left\{\sum_{i=1}^{k} \frac{I_{i}\left(x\left(t_{i}\right)\right)}{E_{\alpha}\left(-t_{i}^{\alpha} \lambda\right)}\right. \\
& \left.-\kappa \int_{0}^{1}(1-s)^{\alpha-1} E_{\alpha, \alpha}\left(-(1-s)^{\alpha} \lambda\right) f(s, x(s), x(\gamma s)) d s\right\} \\
& -E_{\alpha}\left(-t^{\alpha} \lambda\right) \sum_{j=m+1}^{k} \frac{I_{j}\left(x\left(t_{j}\right)\right)}{E_{\alpha}\left(-t_{j}^{\alpha} \lambda\right)} \\
& +\int_{0}^{t}(t-s)^{\alpha-1} E_{\alpha, \alpha}\left(-(t-s)^{\alpha} \lambda\right) f(s, x(s), x(\gamma s)) d s, \\
& t \in\left[t_{m}, t_{m+1}\right), m=0,1,2, \ldots, k,
\end{aligned}
$$

then we show that $T$ has a fixed point, which is a solution of problem (1.3). Letting $G=$ $\sup _{t \in J}|f(t, 0,0)|, G^{*}=\max \left\{\left|I_{i}(0)\right|: i=1,2, \ldots, k\right\}$, we choose

$$
r \geq \frac{\sum_{i=1}^{k} \frac{G^{*}}{\left|E_{\alpha}\left(-t_{i}^{\alpha} \lambda\right)\right|}+\frac{G}{\Gamma(\alpha+1)}}{\frac{\left|1+\kappa E_{\alpha}(-\lambda)\right|}{(2+\kappa)}-\left[\sum_{i=1}^{k} \frac{L_{2}}{\left|E_{\alpha}\left(-t_{i}^{\alpha} \lambda\right)\right|}+\frac{(4 \kappa+2) L_{1}}{(2+\kappa) \Gamma(\alpha+1)}\right]} .
$$

Firstly, we show that $T H_{r} \subset H_{r}$, where $H_{r}=\left\{x \in P C(J, \mathbb{R}):\|x\|_{P C} \leq r\right\}$. It follows from the hypotheses above and Lemma 2.6 that

$$
\begin{aligned}
& |(T x)(t)| \\
& \quad \leq\left|E_{\alpha}\left(-t^{\alpha} \lambda\right)\right| \mid \frac{1}{1+\kappa E_{\alpha}(-\lambda)}\left\{\sum_{i=1}^{k} \frac{I_{i}\left(x\left(t_{i}\right)\right)}{E_{\alpha}\left(-t_{i}^{\alpha} \lambda\right)}\right.
\end{aligned}
$$




$$
\begin{aligned}
& \left.-\kappa \int_{0}^{1}(1-s)^{\alpha-1} E_{\alpha, \alpha}\left(-(1-s)^{\alpha} \lambda\right) f(s, x(s), x(\gamma s)) d s\right\}-\sum_{j=m+1}^{k} \frac{I_{j}\left(x\left(t_{j}\right)\right)}{E_{\alpha}\left(-t_{j}^{\alpha} \lambda\right)} \\
& +\left|\int_{0}^{t}(t-s)^{\alpha-1} E_{\alpha, \alpha}\left(-(t-s)^{\alpha} \lambda\right) f(s, x(s), x(\gamma s)) d s\right| \\
& \leq \frac{1}{\left|1+\kappa E_{\alpha}(-\lambda)\right|}\left\{\sum_{i=1}^{k} \frac{\left|I_{i}\left(x\left(t_{i}\right)\right)\right|}{\left|E_{\alpha}\left(-t_{i}^{\alpha} \lambda\right)\right|}+\frac{\kappa}{\Gamma(\alpha)} \int_{0}^{1}(1-s)^{\alpha-1}|f(s, x(s), x(\gamma s))| d s\right\} \\
& +\sum_{i=1}^{k} \frac{\left|I_{i}\left(x\left(t_{i}\right)\right)\right|}{\left|E_{\alpha}\left(-t_{i}^{\alpha} \lambda\right)\right|}+\frac{1}{\Gamma(\alpha)} \int_{0}^{t}(t-s)^{\alpha-1}|f(s, x(s), x(\gamma s))| d s \\
& \leq \frac{1+\left|1+\kappa E_{\alpha}(-\lambda)\right|}{\left|1+\kappa E_{\alpha}(-\lambda)\right|}\left\{\sum_{i=1}^{k} \frac{\left|I_{i}\left(x\left(t_{i}\right)\right)-I_{i}(0)\right|+G^{*}}{\left|E_{\alpha}\left(-t_{i}^{\alpha} \lambda\right)\right|}\right\} \\
& +\frac{\kappa}{\Gamma(\alpha)\left|1+\kappa E_{\alpha}(-\lambda)\right|} \int_{0}^{1}(1-s)^{\alpha-1}[|f(s, x(s), x(\gamma s))-f(s, 0,0)|+|f(s, 0,0)|] d s \\
& +\frac{1}{\Gamma(\alpha)} \int_{0}^{t}(t-s)^{\alpha-1}[|f(s, x(s), x(\gamma s))-f(s, 0,0)|+|f(s, 0,0)|] d s \\
& \leq \frac{(2+\kappa)}{\left|1+\kappa E_{\alpha}(-\lambda)\right|} \sum_{i=1}^{k} \frac{L_{2} r+G^{*}}{\left|E_{\alpha}\left(-t_{i}^{\alpha} \lambda\right)\right|}+\frac{G}{\Gamma(\alpha+1)\left|1+\kappa E_{\alpha}(-\lambda)\right|}+\frac{G}{\Gamma(\alpha+1)} \\
& +\frac{\kappa}{\Gamma(\alpha)\left|1+\kappa E_{\alpha}(-\lambda)\right|} \int_{0}^{1}(1-s)^{\alpha-1} L_{1}(|x(s)+x(\gamma s)|) d s \\
& +\frac{1}{\Gamma(\alpha)} \int_{0}^{t}(t-s)^{\alpha-1} L_{1}(|x(s)+x(\gamma s)|) d s \\
& \leq \frac{(2+\kappa)}{\left|1+\kappa E_{\alpha}(-\lambda)\right|}\left\{\sum_{i=1}^{k} \frac{L_{2} r+G^{*}}{\left|E_{\alpha}\left(-t_{i}^{\alpha} \lambda\right)\right|}+\frac{G}{\Gamma(\alpha+1)}\right\} \\
& +\frac{2 \kappa L_{1} r}{\Gamma(\alpha+1)\left|1+\kappa E_{\alpha}(-\lambda)\right|}+\frac{2 L_{1} r}{\Gamma(\alpha+1)} \\
& \leq \frac{(2+\kappa)}{\left|1+\kappa E_{\alpha}(-\lambda)\right|}\left\{\sum_{i=1}^{k} \frac{G^{*}}{\left|E_{\alpha}\left(-t_{i}^{\alpha} \lambda\right)\right|}+\frac{G}{\Gamma(\alpha+1)}\right. \\
& \left.+\left[\sum_{i=1}^{k} \frac{L_{2}}{\left|E_{\alpha}\left(-t_{i}^{\alpha} \lambda\right)\right|}+\frac{(4 \kappa+2) L_{1}}{(2+\kappa) \Gamma(\alpha+1)}\right] r\right\} \\
& \leq r \text {. }
\end{aligned}
$$

Secondly, we show that the mapping $T$ is a contraction. Indeed, given any $x, y \in H_{r}$ and each $t \in J$, we obtain

$$
\begin{aligned}
& |(T x)(t)-(T y)(t)| \\
& =\mid \frac{E_{\alpha}\left(-t^{\alpha} \lambda\right)}{1+\kappa E_{\alpha}(-\lambda)}\left\{\sum_{i=1}^{k} \frac{I_{i}\left(x\left(t_{i}\right)\right)-I_{i}\left(y\left(t_{i}\right)\right)}{E_{\alpha}\left(-t_{i}^{\alpha} \lambda\right)}\right. \\
& \left.\quad-\kappa \int_{0}^{1}(1-s)^{\alpha-1} E_{\alpha, \alpha}\left(-(1-s)^{\alpha} \lambda\right)(f(s, x(s), x(\gamma s))-f(s, y(s), y(\gamma s))) d s\right\}
\end{aligned}
$$




$$
\begin{aligned}
& -\sum_{j=m+1}^{k} \frac{I_{j}\left(x\left(t_{j}\right)\right)-I_{j}\left(y\left(t_{j}\right)\right)}{E_{\alpha}\left(-t_{j}^{\alpha} \lambda\right)} \\
& +\int_{0}^{t}(t-s)^{\alpha-1} E_{\alpha, \alpha}\left(-(t-s)^{\alpha} \lambda\right)(f(s, x(s), x(\gamma s))-f(s, y(s), y(\gamma s))) d s \mid \\
\leq & \left(\frac{1}{\left|1+\kappa E_{\alpha}(-\lambda)\right|}+1\right) \sum_{i=1}^{k} \frac{\left.L_{2} \mid x\left(t_{i}\right)\right)-\left(y\left(t_{i}\right)\right) \mid}{E_{\alpha}\left(-t_{i}^{\alpha} \lambda\right)} \\
& +\frac{\kappa}{\Gamma(\alpha)\left|1+\kappa E_{\alpha}(-\lambda)\right|} \int_{0}^{1}(1-s)^{\alpha-1} L_{1}(|x(s)-y(s)|+|x(\gamma s)-y(\gamma s)|) d s \\
& +\frac{1}{\Gamma(\alpha)} \int_{0}^{t}(t-s)^{\alpha-1} L_{1}(|x(s)-y(s)|+|x(\gamma s)-y(\gamma s)|) d s \\
\leq & \frac{(2+\kappa)}{\left|1+\kappa E_{\alpha}(-\lambda)\right|} \sum_{i=1}^{k} \frac{X_{2}}{E_{\alpha}\left(-t_{i}^{\alpha} \lambda\right)}\|x-y\|_{P C} \\
& +\frac{2 \kappa L_{1}}{\Gamma(\alpha+1)\left|1+\kappa E_{\alpha}(-\lambda)\right|}\|x-y\|_{P C}+\frac{2 L_{1}}{\Gamma(\alpha+1)}\|x-y\|_{P C} \\
\leq & \frac{(2+\kappa)}{\left|1+\kappa E_{\alpha}(-\lambda)\right|}\left(\sum_{i=1}^{k} \frac{L_{2}}{E_{\alpha}\left(-t_{i}^{\alpha} \lambda\right)}+\frac{(4 \kappa+2) L_{1}}{(2+\kappa) \Gamma(\alpha+1)}\right)\|x-y\|_{P C} \\
= & \eta\|x-y\|_{P C} .
\end{aligned}
$$

This implies that $\|T x-T y\| \leq \eta\|x-y\|_{P C}$. Thus, $T$ is a contraction, Hence we conclude the proof by applying Banach contraction principle.

Theorem 3.2 Assume that condition $\left(C_{2}\right)$ and the following additional conditions are satisfied:

$\left(C_{4}\right)$ A function $\varphi \in L^{(1 / \rho)}(J,(0,+\infty))(0<\rho<\alpha<1)$ exists, and $\bar{\omega} \in C([0,+\infty])$ is a nondecreasing function satisfying the following inequality:

$$
|f(t, x(s), x(\gamma s))| \leq \varphi(t) \bar{\omega}\left(\|x\|_{P C}\right), \quad x \in P C(J, \mathbb{R}), t \in J .
$$

$\left(C_{5}\right)$

$$
\frac{(2+\kappa)}{\left|1+\kappa E_{\alpha}(-\lambda)\right|}\left(\frac{(1+2 \kappa)\|\varphi\|_{\left.L^{\frac{1}{\rho}}()\right)}}{(2+\kappa) \Gamma(\alpha)\left(\frac{\alpha-\rho}{1-\rho}\right)^{1-\rho}} \liminf _{r \rightarrow+\infty} \frac{\bar{\omega}(r)}{r}+\sum_{i=1}^{k} \frac{L_{2}}{\left|E_{\alpha}\left(-t_{i}^{\alpha} \lambda\right)\right|}\right)<1 .
$$

Then problem (1.3) has at least one solution.

Proof It is easily to see that the set $H_{r}=\left\{x \in P C(J, \mathbb{R}):\|x\|_{P C} \leq r\right\}$ is a closed, bounded, and convex set in $P C(J, \mathbb{R})$ for all $r>0$. Let $M$ and $N$ be two operators on $H_{r}$ defined by

$$
\begin{aligned}
(M x)(t)= & \int_{0}^{t}(t-s)^{\alpha-1} E_{\alpha, \alpha}\left(-(t-s)^{\alpha} \lambda\right) f(s, x(s), x(\gamma s)) d s \\
& -\frac{\kappa E_{\alpha}\left(-t^{\alpha} \lambda\right)}{1+\kappa E_{\alpha}(-\lambda)} \int_{0}^{1}(1-s)^{\alpha-1} E_{\alpha, \alpha}\left(-(1-s)^{\alpha} \lambda\right) f(s, x(s), x(\gamma s)) d s,
\end{aligned}
$$




$$
(N x)(t)=\frac{E_{\alpha}\left(-t^{\alpha} \lambda\right)}{1+\kappa E_{\alpha}(-\lambda)} \sum_{i=1}^{k} \frac{I_{i}\left(x\left(t_{i}\right)\right)}{E_{\alpha}\left(-t_{i}^{\alpha} \lambda\right)}-E_{\alpha}\left(-t^{\alpha} \lambda\right) \sum_{j=m+1}^{k} \frac{I_{j}\left(x\left(t_{j}\right)\right)}{E_{\alpha}\left(-t_{j}^{\alpha} \lambda\right)} .
$$

It follows from condition $\left(C_{4}\right)$ and Hölder inequality that for any $x \in H_{r}$ and each $t \in J$,

$$
\begin{aligned}
& \int_{0}^{t}\left|(t-s)^{\alpha-1} f(s, x(s), x(\gamma s))\right| d s \\
& \quad \leq \int_{0}^{t}\left|(t-s)^{\alpha-1} \varphi(s) \bar{\omega}(r)\right| d s \\
& \quad \leq\left(\int_{0}^{t}(t-s)^{\frac{\alpha-1}{1-\rho}} d s\right)^{1-\rho}\left(\int_{0}^{t}(\bar{\omega}(r) \varphi(s))^{\frac{1}{\rho}} d s\right)^{\rho} \\
& \quad \leq \frac{\|_{L^{\frac{1}{\rho}}}(J)}{\left(\frac{\alpha-\rho}{1-\rho}\right)^{1-\rho}} \bar{\omega}(r) .
\end{aligned}
$$

Repeating the same procedure as above, we obtain

$$
\int_{0}^{1}(1-s)^{\alpha-1} E_{\alpha, \alpha}\left(-(1-s)^{\alpha} \lambda\right) f(s, x(s), x(\gamma s)) d s \leq \frac{\|\varphi\|_{L^{\frac{1}{\rho}}(J)}}{\left(\frac{\alpha-\rho}{1-\rho}\right)^{1-\rho}} \bar{\omega}(r) .
$$

Next, we show that there exist $r_{0}>0$ with $M x+N y \in H_{r_{0}}$ for $x, y \in H_{r_{0}}$. Suppose by contradiction that for each $r>0$ there exist $x_{r}, y_{r} \in H_{r_{0}}$ and $t_{r} \in J$ such that $\left|\left(M x_{r}\right)\left(t_{r}\right)+\left(N y_{r}\right)\left(t_{r}\right)\right|>$ $r$. Assumption $\left(C_{2}\right)$ implies that $\mid I_{i}\left(x\left(t_{i}\right)|\leq| I_{i}\left(x\left(t_{i}\right)-I_{i}(0)+I_{i}(0) \mid \leq L_{2} r+G^{*}\right.\right.$.

Thus,

$$
\begin{aligned}
r< & \left|\left(M x_{r}\right)\left(t_{r}\right)+\left(N y_{r}\right)\left(t_{r}\right)\right| \\
\leq & \frac{\kappa\|\varphi\|_{\left.L^{\frac{1}{\rho}}()\right)}}{\Gamma(\alpha)\left|1+\kappa E_{\alpha}(-\lambda)\right|\left(\frac{\alpha-\rho}{1-\rho}\right)^{1-\rho}} \bar{\omega}(r) \\
& +\frac{\|\varphi\|_{\left.L^{\frac{1}{\rho}}()\right)}}{\Gamma(\alpha)\left(\frac{\alpha-\rho}{1-\sigma}\right)^{1-\rho}} \bar{\omega}(r)+\frac{1}{\left|1+\kappa E_{\alpha}(-\lambda)\right|} \sum_{i=1}^{k} \frac{L_{2} r+G^{*}}{\left|E_{\alpha}\left(-t_{i}^{\alpha} \lambda\right)\right|}+\sum_{i=1}^{k} \frac{L_{2} r+G^{*}}{\left|E_{\alpha}\left(-t_{i}^{\alpha} \lambda\right)\right|} \\
\leq & \frac{(2+\kappa)}{\left|1+\kappa E_{\alpha}(-\lambda)\right|}\left(\frac{(1+2 \kappa)\|\varphi\|_{\left.L^{\frac{1}{\rho}}()\right)}}{(2+\kappa) \Gamma(\alpha)\left(\frac{\alpha-\rho}{1-\rho}\right)^{1-\rho}} \bar{\omega}(r)+\sum_{i=1}^{k} \frac{L_{2} r+G^{*}}{\left|E_{\alpha}\left(-t_{i}^{\alpha} \lambda\right)\right|}\right) .
\end{aligned}
$$

Dividing both sides by $r$ and taking the lower limit as $r \rightarrow+\infty$, yields

$$
1 \leq \frac{(2+\kappa)}{\left|1+\kappa E_{\alpha}(-\lambda)\right|}\left(\frac{(1+2 \kappa)\|\varphi\|_{L^{\frac{1}{\rho}}(J)}}{(2+\kappa) \Gamma(\alpha)\left(\frac{\alpha-\rho}{1-\rho}\right)^{1-\rho}} \liminf _{r \rightarrow+\infty} \frac{\bar{\omega}}{r}+\sum_{i=1}^{k} \frac{L_{2}}{\left|E_{\alpha}\left(-t_{i}^{\alpha} \lambda\right)\right|}\right),
$$

which contradicts condition $\left(C_{5}\right)$. Hence, there exist $r_{0}$ such that $M x+N y \in H_{r_{0}}$, for all $x, y \in H_{r_{0}}$.

Thus, for all $t \in J$ and $x, y \in H_{r}$, one gets

$$
|(N x)(t)-(N y)(t)| \leq \frac{\left|E_{\alpha}\left(-t^{\alpha} \lambda\right)\right|}{\left|1+\kappa E_{\alpha}(-\lambda)\right|} \sum_{i=1}^{k} \frac{I_{i}\left(x\left(t_{i}\right)\right)-I_{i}\left(y\left(t_{i}\right)\right)}{\left|E_{\alpha}\left(-t_{i}^{\alpha} \lambda\right)\right|}
$$




$$
\begin{aligned}
& +\left|E_{\alpha}\left(-t_{i}^{\alpha} \lambda\right)\right| \sum_{i=1}^{k} \frac{I_{i}\left(x\left(t_{i}\right)\right)-I_{i}\left(y\left(t_{i}\right)\right)}{\left|E_{\alpha}\left(-t_{i}^{\alpha} \lambda\right)\right|} \\
\leq & \sum_{i=1}^{k} \frac{I_{i}\left(x\left(t_{i}\right)\right)-I_{i}\left(y\left(t_{i}\right)\right)}{\left|E_{\alpha}\left(-t_{i}^{\alpha} \lambda\right)\right|}\left(1+\frac{1}{\left|1+\kappa E_{\alpha}(-\lambda)\right|}\right) \\
\leq & \left(\frac{(2+\kappa)}{\left|1+\kappa E_{\alpha}(-\lambda)\right|} \sum_{i=1}^{k} \frac{L_{2}}{\left|E_{\alpha}\left(-t_{i}^{\alpha} \lambda\right)\right|}\right)\|x-y\|_{P C} .
\end{aligned}
$$

Denoting $\eta^{*}=\frac{(2+\kappa)}{\left|1+\kappa E_{\alpha}(-\lambda)\right|} \sum_{i=1}^{k} \frac{L_{2}}{\left|E_{\alpha}\left(-t_{T}^{\alpha} \lambda\right)\right|}$, it follows from $\left(C_{5}\right)$ that $0<\eta^{*}<1$ and $\| N x+$ $N y\left\|_{P C} \leq \eta^{*}\right\| x-y \|_{P C}$. Thus $N$ is a contraction mapping.

Since $f$ is continuous, this implies that operator $M$ is also continuous. Now to show $M$ is compact, we apply the same procedure as in Theorem 3.1. One can easily see that $M\left(H_{r}\right)$ is uniformly bounded on $P C(J, \mathbb{R})$. We now show that $M\left(H_{r}\right)$ is equicontinuous on $J_{m}(m=1, \ldots, k)$. Let $\Phi=J \times H_{r} \times H_{r}$ and $f^{*}=\sup _{(t, x(t), x(\gamma s)) \in \Phi}|f(t, x(t), x(\gamma s))|$, then, for any $t_{m}<\xi_{2}<\xi_{1}<t_{m+1}$, we have

$$
\begin{aligned}
& \left|(M x)\left(\xi_{2}\right)-(M x)\left(\xi_{1}\right)\right| \\
& \leq \mid \int_{0}^{\xi_{2}}\left(\xi_{2}-s\right)^{\alpha-1} E_{\alpha, \alpha}\left(-\left(\xi_{2}-s\right)^{\alpha} \lambda\right)(f(s, x(s), x(\gamma s)) d s \\
& -\int_{0}^{\xi_{1}}\left(\xi_{1}-s\right)^{\alpha-1} E_{\alpha, \alpha}\left(-(t-s)^{\alpha} \lambda\right)(f(s, x(s), x(\gamma s)) d s \mid \\
& +\left|\frac{\kappa\left(E_{\alpha}\left(-\tau_{2}^{\alpha} \lambda\right)-E_{\alpha}\left(-\tau_{1}^{\alpha} \lambda\right)\right)}{1+\kappa E_{\alpha}(-\lambda)} \int_{0}^{1}(1-s)^{\alpha-1} E_{\alpha, \alpha}\left(-(1-s)^{\alpha} \lambda\right) f(s, x(s), x(\gamma s)) d s\right| \\
& \leq \mid \int_{0}^{\xi_{2}}\left(\xi_{2}-s\right)^{\alpha-1} E_{\alpha, \alpha}\left(-\left(\xi_{2}-s\right)^{\alpha} \lambda\right)(f(s, x(s), x(\gamma s)) d s \\
& -\int_{0}^{\xi_{2}}\left(\xi_{1}-s\right)^{\alpha-1} E_{\alpha, \alpha}\left(-\left(\xi_{2}-s\right)^{\alpha} \lambda\right)(f(s, x(s), x(\gamma s)) d s \\
& +\int_{0}^{\xi_{2}}\left(\xi_{1}-s\right)^{\alpha-1} E_{\alpha, \alpha}\left(-\left(\xi_{2}-s\right)^{\alpha} \lambda\right)(f(s, x(s), x(\gamma s)) d s \\
& -\int_{0}^{\xi_{2}}\left(\xi_{1}-s\right)^{\alpha-1} E_{\alpha, \alpha}\left(-\left(\tau_{1}-s\right)^{\alpha} \lambda\right)(f(s, x(s), x(\gamma s)) d s \\
& -\int_{\xi_{2}}^{\xi_{1}}\left(\xi_{1}-s\right)^{\alpha-1} E_{\alpha, \alpha}\left(-\left(\xi_{1}-s\right)^{\alpha} \lambda\right)(f(s, x(s), x(\gamma s)) d s \\
& +\frac{\left|\kappa\left(E_{\alpha}\left(-\xi_{2}^{\alpha} \lambda\right)-E_{\alpha}\left(-\xi_{1}^{\alpha} \lambda\right)\right)\right|}{\Gamma(\alpha)\left|1+\kappa E_{\alpha}(-\lambda)\right|} \int_{0}^{1}(1-s)^{\alpha-1}|f(s, x(s), x(\gamma s))| d s \\
& \leq \int_{0}^{\xi_{2}}\left|\left(\xi_{2}-s\right)^{\alpha-1}-\left(\xi_{1}-s\right)^{\alpha-1}\right|\left|E_{\alpha, \alpha}\left(-\left(\xi_{2}-s\right)^{\alpha} \lambda\right)\right| f^{*} d s \\
& +\int_{0}^{\xi_{2}}\left|\left(\xi_{1}-s\right)^{\alpha-1}\right|\left|E_{\alpha, \alpha}\left(-\left(\xi_{2}-s\right)^{\alpha} \lambda\right)-E_{\alpha, \alpha}\left(-\left(\xi_{1}-s\right)^{\alpha} \lambda\right)\right| f^{*} d s \\
& +\frac{f^{*}}{\Gamma(\alpha)}\left|\int_{\xi_{2}}^{\xi_{1}}\left(\xi_{1}-s\right)^{\alpha-1}\right|+\frac{\left|\kappa\left(E_{\alpha}\left(-\xi_{2}^{\alpha} \lambda\right)-E_{\alpha}\left(-\xi_{1}^{\alpha} \lambda\right)\right)\right|}{\Gamma(\alpha+1)\left|1+\kappa E_{\alpha}(-\lambda)\right|} f^{*} \\
& \leq \frac{f^{*}}{\Gamma(\alpha)}\left|\int_{0}^{\xi_{2}}\right|\left(\xi_{2}-s\right)^{\alpha-1}-\left(\xi_{1}-s\right)^{\alpha-1}|d s|+\frac{\left(\xi_{1}-\xi_{2}\right)^{\alpha} f^{*}}{\Gamma(\alpha+1)}
\end{aligned}
$$




$$
\begin{aligned}
& +\frac{\left|\kappa\left(E_{\alpha}\left(-\xi_{2}^{\alpha} \lambda\right)-E_{\alpha}\left(-\xi_{1}^{\alpha} \lambda\right)\right)\right|}{\Gamma(\alpha+1)\left|1+\kappa E_{\alpha}(-\lambda)\right|} f^{*} \\
& +f^{*} \int_{0}^{\xi_{2}}\left|\left(\xi_{1}-s\right)^{\alpha-1}\right|\left|E_{\alpha, \alpha}\left(-\left(\xi_{2}-s\right)^{\alpha} \lambda\right)-E_{\alpha, \alpha}\left(-\left(\xi_{1}-s\right)^{\alpha} \lambda\right)\right| d s \\
& \leq \frac{\left(\xi_{1}-\xi_{2}\right)^{\alpha}+\xi_{1}^{\alpha}-\xi_{2}^{\alpha}}{\Gamma(\alpha+1)} f^{*}+\frac{\left(\xi_{1}-\xi_{2}\right)^{\alpha} f^{*}}{\Gamma(\alpha+1)}+\frac{\left|\kappa\left(E_{\alpha}\left(-\xi_{2}^{\alpha} \lambda\right)-E_{\alpha}\left(-\xi_{1}^{\alpha} \lambda\right)\right)\right|}{\Gamma(\alpha+1)\left|1+\kappa E_{\alpha}(-\lambda)\right|} f^{*} \\
& +f^{*} \int_{0}^{\xi_{2}}\left(\xi_{1}-s\right)^{\alpha-1}\left|E_{\alpha, \alpha}\left(-\left(\xi_{2}-s\right)^{\alpha} \lambda\right)-E_{\alpha, \alpha}\left(-\left(\xi_{1}-s\right)^{\alpha} \lambda\right)\right| d s .
\end{aligned}
$$

By (2) of Lemma 2.6, it follows that $E_{\alpha, \alpha}\left(-t^{\alpha} \lambda\right)$ is continuous on $t \in J$, and thus $E_{\alpha, \alpha}\left(-t^{\alpha} \lambda\right)$ is uniformly continuous on $t \in J$, hence, there is a sufficiently small $\delta>0$ such that, for $t_{1}, t_{2} \in J$ with $\left|t_{1}-t_{2}\right| \leq \delta$, we have

$$
\left|E_{\alpha, \alpha}\left(-t_{1}^{\alpha} \lambda\right)-E_{\alpha, \alpha}\left(-t_{2}^{\alpha} \lambda\right)\right|<\frac{\epsilon}{\xi_{2}^{\frac{\alpha}{2-\alpha}}} .
$$

Let $\rho_{1}=\frac{2-\alpha}{2(1-\alpha)}$ and $\rho_{2}=\frac{2-\alpha}{\alpha}$. Then $\rho_{1}>1, \rho_{2}>1$, and $\frac{1}{\rho_{1}}+\frac{1}{\rho_{2}}=1$. Applying Hölder inequality yields

$$
\begin{aligned}
\int_{0}^{\xi_{2}}\left(\xi_{1}-s\right)^{\alpha-1}\left|E_{\alpha, \alpha}\left(-\left(\xi_{2}-s\right)^{\alpha} \lambda\right)-E_{\alpha, \alpha}\left(-\left(\xi_{1}-s\right)^{\alpha} \lambda\right)\right| d s \\
\leq\left[\int_{0}^{\xi_{2}}\left(\xi_{1}-s\right)^{(\alpha-1) \frac{2-\alpha}{2(1-\alpha)}} d s\right]^{\frac{2(1-\alpha)}{(2-\alpha)}} \\
\quad \times\left[\int_{0}^{\xi_{2}}\left(E_{\alpha, \alpha}\left(-\left(\xi_{2}-s\right)^{\alpha} \lambda\right)-E_{\alpha, \alpha}\left(-\left(\xi_{1}-s\right)^{\alpha} \lambda\right)\right)^{\frac{2-\alpha}{\alpha}} d s\right]^{\frac{\alpha}{2-\alpha}} \\
\leq\left[\frac{2 \xi_{1}^{\frac{\alpha}{2}}-2\left(\xi_{1}-\xi_{2}\right)^{\frac{\alpha}{2}}}{\alpha}\right]^{\frac{2(1-\alpha)}{2-\alpha}} \epsilon .
\end{aligned}
$$

Hence,

$$
\begin{aligned}
& \left|(M x)\left(\xi_{2}\right)-(M x)\left(\xi_{1}\right)\right| \\
& \quad \leq \frac{\left(\xi_{1}-\xi_{2}\right)^{\alpha}+\xi_{1}^{\alpha}-\xi_{2}^{\alpha}}{\Gamma(\alpha+1)} f^{*}+\frac{\left(\xi_{1}-\xi_{2}\right)^{\alpha} f^{*}}{\Gamma(\alpha+1)}+\frac{\left|\kappa\left(E_{\alpha}\left(-\xi_{2}^{\alpha} \lambda\right)-E_{\alpha}\left(-\xi_{1}^{\alpha} \lambda\right)\right)\right|}{\Gamma(\alpha+1)\left|1+\kappa E_{\alpha}(-\lambda)\right|} f^{*} \\
& \quad+\left[\frac{2 \xi_{1}^{\frac{\alpha}{2}}-2\left(\xi_{1}-\xi_{2}\right)^{\frac{\alpha}{2}}}{\alpha}\right]^{\frac{2(1-\alpha)}{2-\alpha}} \epsilon \\
& \quad \rightarrow 0
\end{aligned}
$$

as $\xi_{2} \rightarrow \xi_{1}$, which implies that $M$ is equicontinuous on the interval $J_{m}$. Hence, we have shown that $M\left(H_{r}\right)$ is relatively compact on $J$. It now follows by Arzela-Ascoli's theorem that $M$ is compact. Therefore, we conclude from Lemma 2.7 that problem (1.3) has at least one solution.

We now present some examples to illustrate our result. 
Example 1 Consider the following fractional pantograph-differential equation with impulsive and anti-periodic boundary condition described by:

$$
\left\{\begin{array}{l}
{ }^{c} D_{0+}^{\frac{1}{2}} x(t)+x(t)=\frac{\sin (t)}{(t+5)^{2}} \frac{x^{2}(t)}{\left(1+x^{2}(t)\right)}+\frac{\cos (t)}{\left(e^{t}+4\right)^{2}} x\left(\frac{1}{3} t\right), \quad t \in J \in[0,1] \backslash\left\{\frac{1}{2}\right\} \\
\left.\Delta x\right|_{t=\frac{1}{2}}=\frac{\left|x\left(\frac{1}{2}\right)\right|}{17+\left|x\left(\frac{1}{2}\right)\right|} \\
x(0)=-x(1) .
\end{array}\right.
$$

By comparing with problem (1.3), we get:

$f(t, x(t), x(\gamma t))=\frac{\sin (t)}{(t+5)^{2}} \frac{x^{2}(t)}{\left(1+x^{2}(t)\right)}+\frac{\cos (t)}{\left(e^{t}+4\right)^{2}} x\left(\frac{1}{3} t\right), t \in J \in[0,1] \backslash\left\{\frac{1}{2}\right\}, a=b=\kappa=1, \alpha=\frac{1}{2}, I_{k}(x)=$ $\frac{\left|x\left(\frac{1}{2}\right)\right|}{17+\left|x\left(\frac{1}{2}\right)\right|}$.

Then for any $x, y, u, v \in \mathbb{R}$ and $t \in J$, we obtain

$$
\begin{aligned}
& |f(t, x, y)-f(t, u, v)| \leq \frac{2}{25}\|x-y\|_{P C}, \\
& \left|I_{k}(x)-I_{k}(y)\right| \leq \frac{1}{17}\|x-y\|_{P C} .
\end{aligned}
$$

Then by a simple calculation we can easily see that $L_{1}=\frac{2}{25}, L_{2}=\frac{1}{17}, E_{\frac{1}{2}}(-1) \approx 0.42$, $E_{\frac{1}{2}}\left(-\left(\frac{1}{2}\right)^{\frac{1}{2}}\right) \approx 0.52, \Gamma\left(\frac{3}{2}\right) \approx 0.89$, and

$$
\eta=\frac{3}{\left|1+E_{\frac{1}{2}}(-1)\right|}\left(\frac{L_{2}}{\left|E_{\frac{1}{2}}\left(-\left(\frac{1}{2}\right)^{\frac{1}{2}}\right)\right|}+\frac{6 \times L_{1}}{3 \times \Gamma\left(\frac{3}{2}\right)}\right) \approx \frac{3}{1.42}\left(\frac{\frac{1}{17}}{0.52}+\frac{(6) \frac{2}{25}}{3 \times(0.89)}\right)<1 .
$$

Therefore, all the hypotheses of Theorem 3.1 are satisfied. Hence problem (1.3) has a unique solution on $[0,1]$.

Example 2 Consider the following impulsive fractional pantograph differential equation:

$$
\left\{\begin{array}{l}
{ }^{c} D_{0+}^{\frac{1}{2}} x(t)+2 x(t)=\frac{\sqrt[3]{t+1} \sin (2 t)}{14}\left(\frac{|x(t)|}{|1+| x(t) \mid}\right)+\frac{\sqrt[3]{t+1}}{\left(7 e^{t}\right)^{2}} x\left(\frac{3}{2} t\right), \quad t \in J \in[0,1] \backslash\left\{\frac{1}{2}\right\} \\
\left.\Delta x\right|_{t=\frac{1}{2}}=\frac{\left|x\left(\frac{1}{2}\right)\right|}{15+\left|x\left(\frac{1}{2}\right)\right|} \\
2 x(0)+4 x(1)=0 .
\end{array}\right.
$$

Denote

$$
f(t, x(t), x(\gamma t))=\frac{\sqrt[3]{t+1} \sin (2 t)}{14}\left(\frac{|x(t)|}{|1+| x(t) \mid}\right)+\frac{\sqrt[3]{t+1}}{\left(7 e^{t}\right)^{2}} x\left(\frac{3}{2} t\right) .
$$

This implies that

$$
|f(t, x(t), x(\gamma t))| \leq\left(\frac{\sqrt[3]{t+1}}{14}\right)(\|x(t)\|+1)
$$

Thus, applying the same procedure as above yields $\alpha=\frac{1}{2}, \lambda=2, \rho=\frac{1}{3}, \kappa=2, \bar{\omega}=r+$ $1, E_{\frac{1}{2}}(-2) \approx 0.25, E_{\frac{1}{2}}\left(-2\left(\frac{1}{2}\right)^{\frac{1}{2}}\right) \approx 0.40, \Gamma\left(\frac{1}{2}\right) \approx 3.14$ and $\varphi(t)=\frac{\sqrt[3]{t+1}}{14}$. This implies that 
$\liminf _{r \rightarrow+\infty} \frac{\bar{\omega}}{r}=1, L_{2}=\frac{1}{15}$ and $\|\varphi(t)\|_{L^{\frac{1}{\rho}}}=\left(\int_{0}^{1}\left(\frac{\sqrt[3]{t+1}}{14}\right)^{3} d t\right)^{\frac{1}{3}} \approx 0.08$. Therefore

$$
\frac{4}{\mid 1+E_{\frac{1}{2}}(-2)}\left(\frac{5\left(\int_{0}^{1}\left(\frac{\sqrt[3]{t+1}}{14}\right)^{3} d t\right)^{\frac{1}{3}}}{4 \Gamma\left(\frac{1}{2}\right)\left(\frac{\frac{1}{2}-\frac{1}{3}}{1-\frac{1}{3}}\right)^{1-\frac{1}{3}}}+\frac{\frac{1}{15}}{\left|E_{\frac{1}{2}}\left(-2\left(\frac{1}{2}\right)^{\frac{1}{2}}\right)\right|}\right) \approx 0.58<1 .
$$

Thus, according to Theorem 3.2, problem (1.3) has at least one solution.

\section{Conclusions}

Using the Banach and Krasnoselskii's fixed point theorems, we have established the existence and uniqueness of the solution for fractional pantograph differential equation with impulsive and generalized anti-periodic boundary conditions. We note that it would be interesting to study this kind of problem for a certain kind of generalized fractional derivatives and integrals [7, 25]. In addition, this is the first paper, to the best of our knowledge, dealing with a fractional pantograph differential equation with an impulsive and generalized anti-periodic boundary conditions. Therefore, our result improves and generalizes the results in [13] and can be considered as a contribution to the development of the qualitative analysis of fractional differential equations. Lastly, we offered two examples to illustrate the obtained results.

\footnotetext{
Acknowledgements

The authors acknowledge the financial support provided by the Center of Excellence in Theoretical and Computational Science (TaCS-CoE), KMUTT. The first and third authors were supported by the "Petchra Pra Jom Klao Ph.D. Research Scholarship from King Mongkut's University of Technology Thonburi". (For Ph.D. Petchra Pra Jom Klao Doctoral Scholarship) (Grant No. 13/2561). Moreover, this research work was financially supported by King Mongkut's University of Technology Thonburi through the KMUTT 55 th Anniversary Commemorative Fund. The authors would also like to thank the anonymous referees for their important comments and valuable feedback that improved manuscript readability.

\section{Funding}

Petchra Pra Jom Klao Doctoral Scholarship for Ph.D. program of King Mongkut's University of Technology Thonburi (KMUTT). The Center of Excellence in Theoretical and Computational Science (TaCS-CoE), KMUTT. King Mongkut's University of Technology North Bangkok, Contract no. KMUTNB-63-KNOW-033.
}

\section{Availability of data and materials}

The sharing of data does not apply to this article since no data sets were produced or analyzed during the current study.

\section{Competing interests}

The authors declare no conflict of interest.

\section{Authors' contributions}

The authors contributed equally in writing this article. All authors read and approved the final manuscript.

\section{Author details}

${ }^{1}$ KMUTTFixed Point Research Laboratory, Room SCL 802 Fixed Point Laboratory, Science Laboratory Building, Department of Mathematics, Faculty of Science, King Mongkut's University of Technology Thonburi (KMUTT), 126 Pracha-Uthit Road, Bang Mod, Thrung Khru, Bangkok, 10140, Thailand. ${ }^{2}$ Center of Excellence in Theoretical and Computational Science (TaCS-CoE), Science Laboratory Building, King Mongkut's University of Technology Thonburi (KMUTT), 126 Pracha-Uthit Road, Bang Mod, Thrung Khru, Bangkok, 10140, Thailand. ${ }^{3}$ Department of Mathematics and Computer Science, Faculty of Natural and Applied Sciences, Sule Lamido University, P.M.B 048, Kafin-Hausa, Jigawa State, Nigeria. ${ }^{4}$ Department of Mathematics, Faculty of Sciences, Usmanu Danfodiyo University Sokoto, P.M.B 2346, Sokoto, Nigeria. ${ }^{5}$ Department of Medical Research, China Medical University Hospital, China Medical University, Taichung, 40402, Taiwan. ${ }^{6}$ Intelligent and Nonlinear Dynamic Innovations Research Center, Department of Mathematics, Faculty of Applied Science, King Mongkut's University of Technology North Bangkok (KMUTNB), Wongsawang, Bangsue, Bangkok, 10800, Thailand.

\section{Publisher's Note}

Springer Nature remains neutral with regard to jurisdictional claims in published maps and institutional affiliations. 


\section{References}

1. Agarwal, P.: Some inequalities involving Hadamard-type k-fractional integral operators. Math. Methods Appl. Sci. 40(11), 3882-3891 (2017)

2. Agarwal, P., Al-Mdallal, Q., Cho, Y.J., Jain, S.: Fractional differential equations for the generalized Mittag-Leffler function. Adv. Differ. Equ. 2018, 58 (2018)

3. Agarwal, P., Dragomir, S.S., Jleli, M., Samet, B.: Advances in Mathematical Inequalities and Applications. Springer, Berlin (2018)

4. Agarwal, P., Jain, S., Mansour, T.: Further extended Caputo fractional derivative operator and its applications. Russ. J. Math. Phys. 24(4), 415-425 (2017)

5. Agarwal, R.P., Ahmad, B., Alsaedi, A.: Fractional-order differential equations with anti-periodic boundary conditions: a survey. Bound. Value Probl. 2017(1), 1 (2017)

6. Ahmed, I., Kumam, P., Jarad, F., Borisut, P., Sitthithakerngkiet, K., Ibrahim, A.: Stability analysis for boundary value problems with generalized nonlocal condition via Hilfer-Katugampola fractional derivative. Adv. Differ. Equ. 2020(1), $1(2020)$

7. Ahmed, I., Kumam, P., Shah, K., Borisut, P., Sitthithakerngkiet, K., Demba, M.A.: Stability results for implicit fractional pantograph differential equations via $\phi$-Hilfer fractional derivative with a nonlocal Riemann-Liouville fractional integral condition. Mathematics 8(1), 94 (2020)

8. Aimene, D., Baleanu, D., Seba, D.: Controllability of semilinear impulsive Atangana-Baleanu fractional differential equations with delay. Chaos Solitons Fractals 128, 51-57 (2019)

9. Ali, A., Shah, K., Baleanu, D.: Ulam stability results to a class of nonlinear implicit boundary value problems of impulsive fractional differential equations. Adv. Differ. Equ. 2019, 5 (2019)

10. Anguraj, A., Ravikumar, K., Baleanu, D.: Approximate controllability of a semilinear impulsive stochastic system with nonlocal conditions and Poisson jumps. Adv. Differ. Equ. 2020(1), 65 (2020)

11. Annamalai, A., Kandasamy, B., Baleanu, D., Arumugam, V.: On neutral impulsive stochastic differential equations with Poisson jumps. Adv. Differ. Equ. 2018, 290 (2018)

12. Atanacković, T.M., Pilipović, S., Stanković, B., Zorica, D.: Fractional Calculus with Applications in Mechanics. Wiley Online Library, New York (2014)

13. Balachandran, K., Kiruthika, S., Trujillo, J.: Existence of solutions of nonlinear fractional pantograph equations. Acta Math. Sci. 33(3), 712-720 (2013)

14. Bazgir, H., Ghazanfari, B.: Existence of solutions for fractional integro-differential equations with non-local boundary conditions. Math. Comput. Appl. 23(3), 36 (2018)

15. Belmekki, M., Nieto, J., Rodriguez-Lopez, R.: Existence of solution to a periodic boundary value problem for a nonlinear impulsive fractional differential equation. Electron. J. Qual. Theory Differ. Equ. 2014, 16 (2014)

16. Borisut, P., Kumam, P., Ahmed, I., Sitthithakerngkiet, K.: Nonlinear Caputo fractional derivative with nonlocal Riemann-Liouville fractional integral condition via fixed point theorems. Symmetry 11(6), 829 (2019)

17. Choi, J., Agarwal, P.: A note on fractional integral operator associated with multiindex Mittag-Leffler functions. Filomat 30(7), 1931-1939 (2016)

18. Derfel, G., Iserles, A.: The pantograph equation in the complex plane. J. Math. Anal. Appl. 213(1), 117-132 (1997)

19. Ergören, H., Kılıçman, A.: Non-local boundary value problems for impulsive fractional integro-differential equations in Banach spaces. Bound. Value Probl. 2012(1), 145 (2012)

20. Fec, M., Zhou, Y., Wang, J., et al.: On the concept and existence of solution for impulsive fractional differential equations. Commun. Nonlinear Sci. Numer. Simul. 17(7), 3050-3060 (2012)

21. Gaul, L., Klein, P., Kemple, S.: Damping description involving fractional operators. Mech. Syst. Signal Process. 5(2), 81-88 (1991)

22. Iserles, A.: On the generalized pantograph functional-differential equation. Eur. J. Appl. Math. 4(1), 1-38 (1993)

23. Iserles, A., Liu, Y.: On pantograph integro-differential equations. J. Integral Equ. Appl. 6(2), 213-237 (1994)

24. Jain, S., Agarwal, P., Kilicman, A.: Pathway fractional integral operator associated with 3m-parametric Mittag-Leffle functions. Int. J. Appl. Comput. Math. 5, 115 (2018)

25. Jarad, F., Abdeljawad, T:: A modified Laplace transform for certain generalized fractional operators. Results Nonlinear Anal. 1(2), 88-98 (2018)

26. Kilbas, A.: Theory and applications of fractional differential equations

27. Krasnoselskii, M.: Two remarks about the method of successive approximations. Usp. Mat. Nauk 10, 123-127 (1955)

28. Li, B., Gou, H.: Existence of solutions for impulsive fractional evolution equations with periodic boundary condition. Adv. Differ. Equ. 2017, 236 (2017)

29. Li, D., Liu, M.: Runge-Kutta methods for the multi-pantograph delay equation. Appl. Math. Comput. 163(1), 383-395 (2005)

30. Li, X., Chen, F., Li, X.: Generalized anti-periodic boundary value problems of impulsive fractional differential equations. Commun. Nonlinear Sci. Numer. Simul. 18(1), 28-41 (2013)

31. Liu, M., Li, D.: Properties of analytic solution and numerical solution of multi-pantograph equation. Appl. Math. Comput. 155(3), 853-871 (2004)

32. Liu, Y.: Survey and new results on boundary-value problems of singular fractional differential equations with impulse effects. Electron. J. Differ. Equ. 2016, 296 (2016)

33. Magin, R.L.: Fractional Calculus in Bioengineering, vol. 2. Begell House, Redding (2006)

34. Podlubny, I.: Fractional Differential Equations: An Introduction to Fractional Derivatives, Fractional Differential Equations, to Methods of Their Solution and Some of Their Applications. Elsevier, Amsterdam (1998)

35. Rudolf, H.: Applications of Fractional Calculus in Physics. World Scientific, Singapore (2000)

36. Samko, S.G., Kilbas, A.A., Marichev, O.I., et al.: Fractional Integrals and Derivatives, vol. 1. Gordon \& Breach, Switzerland (1993)

37. Sezer, M., Sahin, N., et al.: Approximate solution of multi-pantograph equation with variable coefficients. J. Comput. Appl. Math. 214(2), 406-416 (2008)

38. Sousa, J.V.D.C., Oliveira, D.D.S., Capelas de Oliveira, E.: On the existence and stability for noninstantaneous impulsive fractional integrodifferential equation. Math. Methods Appl. Sci. 42(4), 1249-1261 (2019) 
39. Srivastava, H., Agarwal, P., Jain, S.: Generating functions for the generalized Gauss hypergeometric functions. Appl. Math. Comput. 247, 348-352 (2014)

40. Stamova, I., Stamov, G.: Functional and Impulsive Differential Equations of Fractional Order: Qualitative Analysis and Applications. CRC Press, Boca Raton (2017)

41. Suganya, S., Mallika Arjunan, M.: Existence of mild solutions for impulsive fractional integro-differential inclusions with state-dependent delay. Mathematics 5(1), 9 (2017)

42. Tian, Y., Bai, Z: Impulsive boundary value problem for differential equations with fractional order. Differ. Equ. Dyn. Syst. 21(3), 253-260 (2013)

43. ur Rehman, M., Eloe, P.W.: Existence and uniqueness of solutions for impulsive fractional differential equations. Appl. Math. Comput. 224, 422-431 (2013)

44. Wang, G., Ahmad, B., Zhang, L., Nieto, J.J.: Comments on the concept of existence of solution for impulsive fractional differential equations. Commun. Nonlinear Sci. Numer. Simul. 19(3), 401-403 (2014)

45. Wang, J., Feckan, M., Zhou, Y.: Presentation of solutions of impulsive fractional Langevin equations and existence results. Eur. Phys. J. Spec. Top. 222(8), 1857-1874 (2013)

46. Wang, J., Fečkan, M., Zhou, Y.: A survey on impulsive fractional differential equations. Fract. Calc. Appl. Anal. 19(4), 806-831 (2016)

47. Wang, J., Lin, Z: On the impulsive fractional anti-periodic BVP modelling with constant coefficients. J. Appl. Math. Comput. 46(1-2), 107-121 (2014)

48. Wei, W., Xiang, X., Peng, Y.: Nonlinear impulsive integro-differential equations of mixed type and optimal controls. Optimization 55(1-2), 141-156 (2006)

49. Wu, G.-C., Baleanu, D., Huang, L.-L.: Novel Mittag-Leffler stability of linear fractional delay difference equations with impulse. Appl. Math. Lett. 82, 71-78 (2018)

50. Wu, G.-C., Zeng, D.-Q., Baleanu, D.: Fractional impulsive differential equations: exact solutions, integral equations and short memory case. Fract. Calc. Appl. Anal. 22(1), 180-192 (2019)

51. Yang, L., Chen, H.: Nonlocal boundary value problem for impulsive differential equations of fractional order. Adv. Differ. Equ. 2011, 404917 (2011)

52. Yang, S., Zhang, S.: Boundary value problems for impulsive fractional differential equations in Banach spaces. Filomat $31(18), 5603-5616(2017)$

53. Yu, Z.-H.: Variational iteration method for solving the multi-pantograph delay equation. Phys. Lett. A 372(43), 6475-6479 (2008)

54. Zhao, K., Gong, P.: Positive solutions for impulsive fractional differential equations with generalized periodic boundary value conditions. Adv. Differ. Equ. 2014, 255 (2014)

55. Zou, Y., Cui, Y.: Existence results for a functional boundary value problem of fractional differential equations. Adv. Differ. Equ. 2013, 233 (2013)

56. Zuo, M., Hao, X., Liu, L., Cui, Y.: Existence results for impulsive fractional integro-differential equation of mixed type with constant coefficient and antiperiodic boundary conditions. Bound. Value Probl. 2017(1), 1 (2017)

\section{Submit your manuscript to a SpringerOpen ${ }^{\circ}$ journal and benefit from:}

- Convenient online submission

- Rigorous peer review

- Open access: articles freely available online

- High visibility within the field

- Retaining the copyright to your article

Submit your next manuscript at $\gg$ springeropen.com 